\title{
LINEAR EVOLUTION OF SMALL SCALE COSMOLOGICAL DENSITY PERTURBATIONS
}

\author{
K. YAMAMOTO \\ Department of Physics, Hiroshima University, \\ Higashi-Hiroshima 739, Japan \\ AND \\ N. SUGIYAMA AND H. SATO \\ Department of Physics, Kyoto University, Kyoto 606-01, Japan
}

We carefully re-examine the evolution of small scale cosmological perturbations, motivated from the studies of cosmic structure formation in the high- $z$ universe. Under the assumption of the hierarchical clustering scenario, the evolution of density fluctuations on very small scales is especially important for the early formation of the bound objects such as population III stars or primordial sub-galaxies.

Through the interaction with photons via electrons, baryon perturbations show characteristic behavior in some physical scales [1, 2]. In CDM models, it is found a power-law growing phase of the small-scale baryon density fluctuations, which is characterized by the terminal velocity, after the diffusion (Silk) damping and before the decoupling epoch. During this phase, the baryon fluctuations evolve keeping the balance of the gravitational force due to the potential of CDM fluctuations and the friction force due to the interaction with background photons.

Then, a transfer function for total matter density fluctuations is studied by taking into account those physical processes. An analytic transfer function is presented, which is applicable for the entire range up to a solar mass scale in the high $-z$ universe, and it is suitable also to the high baryon fraction models [2].

\section{References}

1. Yamamoto, K., Sugiyama, N. and Sato, H. (1997) Physical Review D, in press, astro-ph/9709209.

2. Yamamoto, K., Sugiyama, N. and Sato, H. (1997) submitted to Astrophysical Journal, astro-ph/9709247. 